\title{
A COMPARAÇÃO TEÓRICA DAS ELETROSSÍNTESE CATÓDICA E ANÓDICA DE POLIPIRROL EM SOLUÇÕES FORTEMENTE ÁCIDAS. CASO DO MODO GALVANOSTÁTICO
}

\author{
V.V, Tkach ${ }^{1 *}$, R. Ojani ${ }^{2}$, C. F. Dallaqua Hirschmann ${ }^{3}$, M. O. Galeano Espínola ${ }^{4}$ O. \\ Yelenich $^{1}$, P. Yagodynets' ${ }^{1}$ \\ ${ }^{1}$ Universidade Nacional de Chernivtsi, Ucrânia \\ ${ }^{2}$ Universidade de Mazandarã, República Islâmica do Irã \\ ${ }^{3}$ Escola Bahiana de Medicina, Salvador, Bahia, Brasil \\ ${ }^{4}$ Faculdade de Ciências Químicas, Universidade Nacional de Assunção, República do \\ Paraguai
}

\section{RESUMO}

Por meio de uma modelagem matemática, foram comparadas as duas vias de eletrossíntese de polipirrol - a catódica e a anódica. Em termos da diferença dos mecanismos de eletrossíntese de polipirrol, bem como dos modelos matemáticos, foi explicada a discrepância entre as estruturas dos polipirróis anódico e catódico, obtidos em meio fortemente ácido no modo galvanostático.

Palavras-chave: polímeros condutores, polipirrol, eletrodeposição, eletropolimerização, estado estacionário estável

\section{INTRODUÇÃO}

Polipirrol [1] é um dos polímeros condutores mais usados, haja vista o seu preço baixo, facilidade de síntese (seja química, ou eletroquímica), modificação e flexibilidade de uso. A eletrossíntese de polipirrol, usualmente, se dá pela eletropolimerização anódica [2], que segue o mecanismo clássico de Díaz.

São poucos os processos catódicos que levam à eletrodeposição de polímeros condutores [3]. Envolvem-se, geralmente, os processos da polimerização indireta, na qual no cátodo se formam, in situ, as partículas oxidantes. Um dos exemplos para polipirrol é o descrito em [4]. Nele, a partícula oxidante foi o nitroso-cátion, formado pela reação:

$$
\mathrm{NO}_{3}+4 \mathrm{H}^{+}+2 \mathrm{e}^{-} \rightarrow \mathrm{NO}^{+}+2 \mathrm{H}_{2} \mathrm{O}
$$

Este cátion, posteriormente, oxida o pirrol até o seu radical-cátion que cresce: 


$$
(2 \mathrm{n}-2) \mathrm{NO}^{+}+\mathrm{nC}_{4} \mathrm{H}_{5} \mathrm{~N} \rightarrow \mathrm{C}_{4} \mathrm{H}_{4} \mathrm{~N}-\left(\mathrm{C}_{4} \mathrm{H}_{3} \mathrm{~N}\right)_{\mathrm{n}-2}-\mathrm{C}_{4} \mathrm{H}_{4} \mathrm{~N}+(2 \mathrm{n}-2) \mathrm{NO}+(2 \mathrm{n}-2) \mathrm{H}^{+}
$$

No mesmo trabalho foram mostradas as imagens de microscopia eletrônica de varredura (MEV) que demonstram que o polipirrol catódico, obtido de uma solução ácida tem uma estrutura mais desenvolvida que obtido por meio de eletropolimerização anódica (Fig. 1, à esquerda):
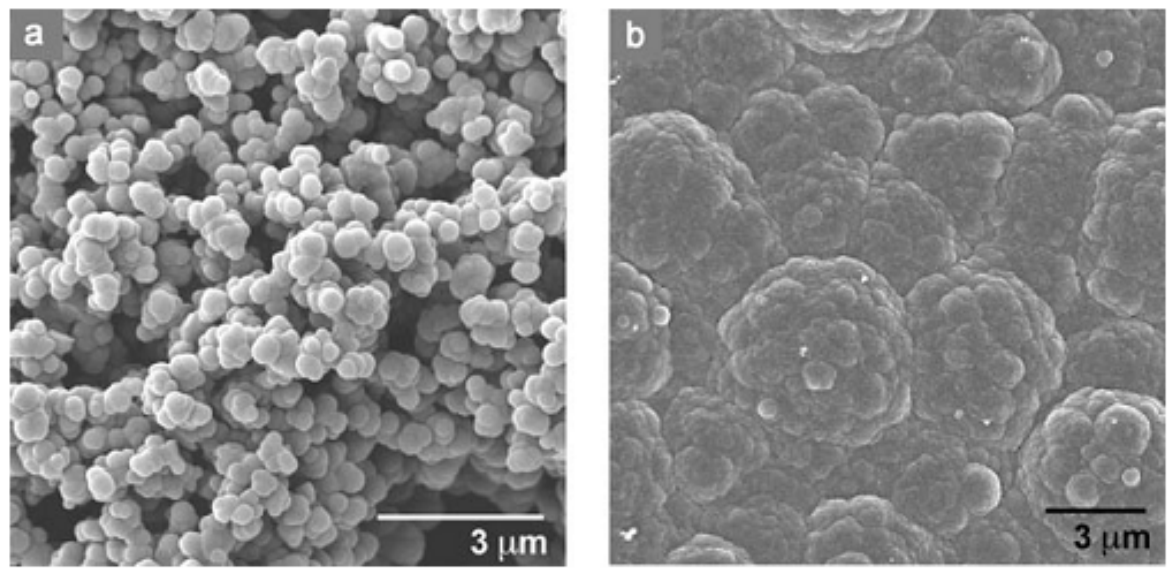

Fig. 1. As imagens de MEV do polipirrol, obtido da solução de 0, 2 M Py + 0,4 M $\mathrm{HNO}_{3} \mathrm{~m}+$ 0,5 $\mathrm{M} \mathrm{NaNO}_{3}$, por meio da eletrodeposição catódica (à esquerda) e anódica (à direita)

Estas diferenças estruturais só podem ser causadas por diferenças detalhadas no mecanismo da eletrossíntese no primeiro e no segundo caso. Buscar-se-ão, neste trabalho, as divergências nos mecanismos de eletrossíntese, por meio da comparação analítica de modelos matemáticos, correspondentes a ambos os casos.

\section{O SISTEMA E O MODELO}

Durante a eletrossíntese galvanostática catódica de polipirrol em soluções muito fortemente ácidas $(\mathrm{pH}<<1)$, os prótons estão em excesso. Além disso, os íons nitrato também estão em concentração excessiva. Introduzir-se-ão, destarte, para a descrição matemática do sistema, as três variáveis:

N - a concentração dos cátions nitroso na camada pré-superficial;

c - a concentração das moléculas de pirrol na camada pré-superficial;

q - a carga do cátodo.

É possível mostrar que, haja vista algumas suposições [5], o sistema se descreve pelo seguinte modelo: 


$$
\left\{\begin{array}{c}
\frac{d N}{d t}=\frac{2}{\delta}\left(r_{i}-r_{p}\right) \\
\frac{d P}{d t}=\frac{2}{\delta}\left(\frac{\Delta}{\delta}\left(P_{b}-P\right)-r_{p}\right) \\
\frac{d q}{d t}=i-i_{F}
\end{array}\right.
$$

em que $\delta$ é espessura da camada pré-superficial, $\mathrm{r}_{\mathrm{i}}$, a velocidade da formação do cátion iniciador, que, haja vista o excesso do nitrato e dos prótons, pode ser descrita como reação de ordem zero, $\mathrm{r}_{\mathrm{p}}$ é a velocidade de polimerização, $\mathrm{P}_{\mathrm{b}}$ é a concentração de pirrol no interior da solução, i é a corrente, aplicada ao cátodo e i F a corrente de Faraday.

As velocidades das reações correspondentes podem ser descritas como:

$$
r_{i}=k_{i} \exp \left(\frac{2 F \varphi_{0}}{R T}\right), \quad r_{p}=k_{p} N^{x} P^{y} \quad i_{F}=2 F k_{i} \exp \left(\frac{2 F \varphi_{0}}{R T}\right)
$$

Em que os parâmetros k são constantes das respectivas reações, x e y são as ordens das respectivas reações por nitrosil-cátions e pirrol, F é o número de Faraday, $\varphi_{0}$ é o salto do potencial, relativo ao potencial de carga zero, $\mathrm{R}$ é a constante universal de gases e $\mathrm{T}$, a temperatura absoluta do vaso.

\section{RESULTADOS E DISCUSSÃO}

Para analisar o comportamento do sistema com a eletrodeposição catódica galvanostática de polipirrol de uma solução fortemente ácida, analisamos o conjunto de equações diferenciais (3), tendo em conta as relações algébricas (4 - 6), mediante a teoria de estabilidade linear. A matriz funcional de Jacobi, cujos membros são calculados para o estado estacionário, pode ser descrita como:

$$
\left(\begin{array}{lll}
a_{11} & a_{12} & a_{13} \\
a_{21} & a_{22} & a_{23} \\
a_{31} & a_{32} & a_{33}
\end{array}\right)
$$

em que:

$$
\begin{array}{lr}
a_{11}=-\frac{2}{\delta} x k_{p} N^{x-1} P^{y}, & a_{12}=-\frac{2}{\delta}\left(y k_{p} N^{x} P^{y-1}\right) \quad a_{13}= \\
-\frac{2}{\delta}\left(k_{i} j \exp \left(\frac{2 F \varphi_{0}}{R T}\right)\right) & a_{22}=-\frac{2}{\delta}\left(\frac{\Delta}{\delta}+y k_{p} N^{x} P^{y-1}\right) \\
a_{21}=-\frac{2}{\delta} x k_{p} N^{x-1} P^{y} & \\
a_{23}=0 &
\end{array}
$$




$$
a_{31}=0 a_{32}=0 a_{33}=-2 F\left(k_{i} j \exp \left(\frac{2 F \varphi_{0}}{R T}\right)\right)
$$

A nulidade dos elementos a31 e a32 explica-se pelo não participarem os próprios nitrosocátions (bem como as moléculas de pirrol) da sua formação

Estabilidade de estado estacionário: Utilizando o critério de Routh-Gurwitz para sistemas tridimensionais, introduzimos novas variáveis, para o jacobiano não ter expressões complexas. Assim, o determinante da matriz reescrever-se-á como:

$$
\frac{2 F}{\delta^{2}}\left|\begin{array}{ccc}
-\Sigma & -J & -K \\
-\Sigma & -k-J & 0 \\
0 & 0 & -K
\end{array}\right|
$$

e a condição de estabilidade Det $\mathrm{J}<0$ obtém-se sob a forma de:

$$
-K \Sigma k<0
$$

que se satisfaz sempre, pois tanto o parâmetro de polimerização $\Sigma$, como o da difusão do pirrol $k$ e o da reação da formação do iniciador $K$, têm valores positivos. Isto vai ao encontro da observação, feita em [2], bem como das feitas em [1, 6 - 14], em que, durante a eletropolimerização anódica, o $\mathrm{pH}$ baixo não favorecia a estabilidade do estado estacionário. Isto também foi confirmado matematicamente em [15 - 21].

Como a difusão de pirrol é um processo mais devagar que os da formação e da polimerização, pode-se afirmar que a reação é controlada pela difusão. A instabilidade monotônica, neste caso, não existe.

Típica para a eletropolimerização anódica [6 - 14], a instabilidade oscilatória (Fig. 2)

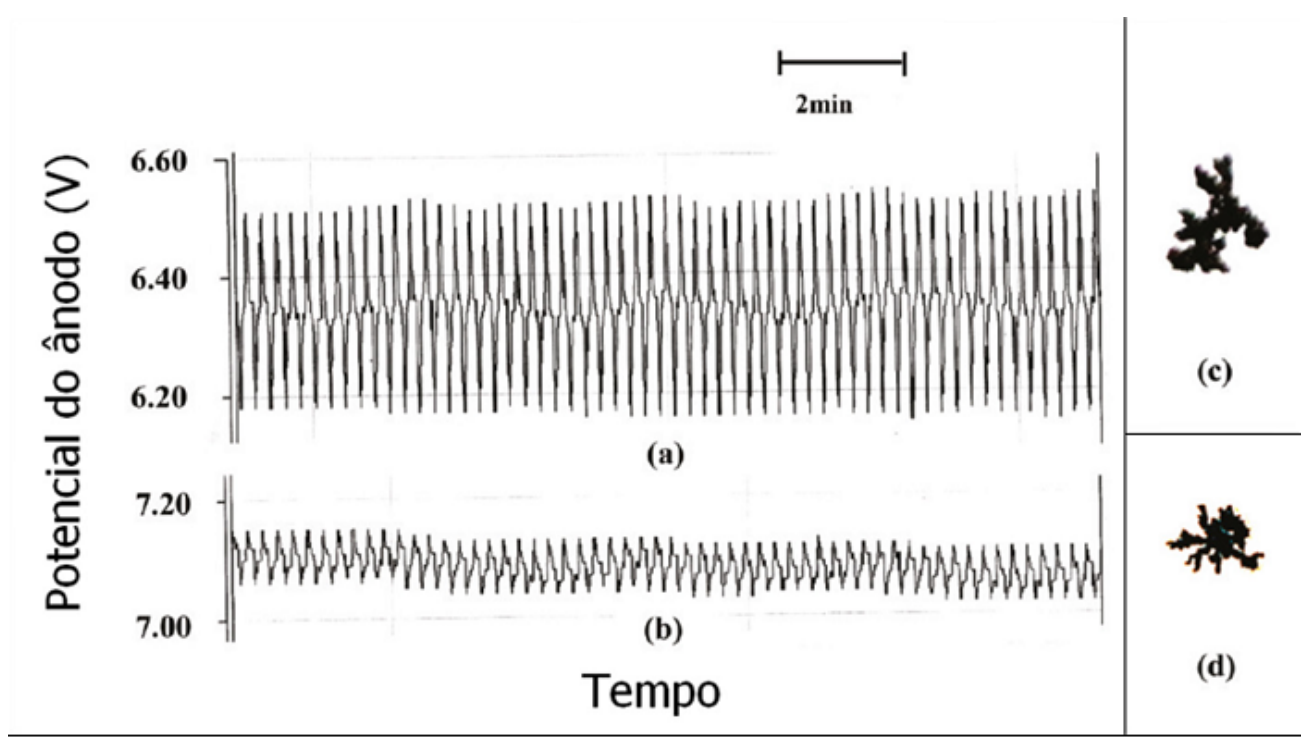

Fig.2. Instabilidade oscilatória, observada durante a eletropolimerização anódica de pirrol em meio fortemente ácido com (a) e sem (b) surfactantes e alterações superficiais correspondentes [6]. 
é impossível para a eletrodeposição catódica. A bifurcação de Hopf, que define o comportamento oscilatório (e é confirmada para a eletropolimerização anódica em [15 21]) requer que na diagonal principal de jacobiano haja elementos de signo positivo, responsáveis pela positiva conexão de retorno. Como não os há, e ainda tendo em vista as observações acima expostas, não pode haver comportamento oscilatório neste caso.

Autocatálise durante a formação de prótons, neste caso, não é possível, haja vista que, quando o nitrato está em excesso, ele reage com os prótons, formando o nitrosil-cátion, que age como iniciador. Este excesso também minimiza, aliás, impede a "evolução de hidrogênio”.

\section{CONCLUSÃO}

1. A eletrodeposição catódica é a maneira mais efetiva de eletrossíntese de polipirrol em soluções ácidas, com excesso de nitrato que a eletropolimerização anódica.

2. Contrariamente ao caso da eletropolimerização, durante a eletrodeposição catódica a diminuição do $\mathrm{pH}$ favorece à estabilidade do estado estacionário. A reação é controlada pela difusão do monômero, que, neste caso, é pirrol.

3. As instabilidades oscilatória e monotônica, contrariamente ao caso da eletropolimerização, não são possíveis para este caso, haja vista a não satisfação das suas condições neste sistema. Destarte, não há, nem pode haver, estruturas dissipativas temporais e espaciais, que poderiam afetar diretamente 0 comportamento deste sistema, o que confirma o experimento.

\section{REFERÊNCIAS}

1. S. Sadki, P. Schottland, N. Brodie, G. Saboraud, Chem. Soc. Rev., 29(2000), 283

2. V.M. de Andrade, Confecção de biossensores através da imobilização de biocomponentes por eletropolimerização de pirrol Tés. M. Eng. UFRGS., Porto Alegre, 2006

3. J. Roncali, Chem. Rev., 92(1992), 711

4. Y.Jung, N. Singh, K.-Sh. Choi, Angew. Chem. Int. Ed., 48(2009), 8331

5. Koper M.T.M. "Far-from-equilibrium phenomena in electrochemical systems: oscillations, instabilities and chaos. -Ph. D. Thesis. Utrecht" Universiteit Utrecht 1994.

6. I.Das, N. Goel, N.R. Agrawal, S. K. Gupta, J. Phys. Chem, 114(2010), 114

7. I.Das, N.R.Agrawal, S.A.Ansari, S.K.Gupta, 47A(2008), 1798 
8. Sazou D., Synth. Met.,130(2002), 45

9. M.S. Ba-Shammakh. "Electropolymerization of pyrrole on mild steel for corrosion protection”, Thes. Ph. D. King Fahd University of Petroleum and Minerals, Dharan, Saudi Arabia, 2002

10. K.R. Lemos Castagno K.R., "Eletropolimerização de pirrol sobre liga de alumínio 1100”, Tés. D. Sc. UFRGS., Porto Alegre, 2007

11. M. Bazzaoui, E.A. Bazzaoui, L. Martins, J.I. Martins, Synth. Met., 130(2002),73

12. I.Das, N. Goel, S. K. Gupta, N.R. Agrawal, J. Electroanal. Chem, 670(2012), 1

13. K.Aoki, I. Mukoyama, J.Chen, Russ.J. Electrochem, 40(2004), 319

14. A.S.Liu, M.A.S. Oliveira., J. Braz. Chem. Soc., 18(2007), 143

15. V. Tkach, V. Nechyporuk, P. Yagodynets', Med. J. Chem., 3(2015), 1122

16. V. Tkach, S. C. de Oliveira, O. I. Aksimentyeva et. al., Mor. J. Chem., 3(2015), 550

17. V. Tkach, V. Nechyporuk, P. Yagodynet', Afinidad, 70(2013), 73

18. V. Tkach, V. Nechyporuk, P. Yagodynets', Ciên. Tecn. Mat., 24(2012), 54

19. V. Tkach, V. Nechyporuk, P. Yagodynets', Al. M. da Rocha, Quím. Mat., 4(2014), 75

20. V. Tkach, V. Nechyporuk, P. Yagodynets', Quím. Bras., 7(2013), 59

21. V. Tkach, V. Nechyporuk, P. Yagodynets', Avan. Quím., 8(2013), 9 